\title{
The Review of Reliability Factors Related to Industrial Robots
}

\author{
Adrian Kampa* \\ Silesian University of Technology, Poland
}

Submission: October 29, 2018; Published: November 06, 2018

*Corresponding author: Adrian Kampa, Institute of Engineering Processes Automation and Integrated Manufacturing Systems, Silesian University of Technology, Poland

\begin{abstract}
Although, the problem of industrial robot reliability is closely related to machine reliability and is well known and described in the literature, it is also more complex and connected with safety requirements and specific robot related problems (near failure situations, human errors, software failures, calibration, singularity, etc.).Compared to the first robot generation, the modern robots are more advanced, functional and reliable. Some robot's producers declare very high robot working time without failures, but there are fewer publications about the real robot reliability and about occurring failures. Some surveys show that not every robot user has monitoring and collects data about robot failures. The practice show, that the most unreliable components are in the robot's equipment, including grippers, tools, sensors, wiring, which are often custom made for different purposes. The lifecycle of a typical industrial robot is about 10-15 years, because the key mechanical components (e.g. drives, gears, bearings) are wearing out. The key factor is the periodical maintenance following the manufacturer's recommendations. After that time, a refurbishment of the robot is possible, and it can work further, but there are also new and better robots from modern generation.
\end{abstract}

Keywords: Industrial robot;Reliability; Failures; Availability; Maintenance; Safety; MTTF; MTBF; MTTR; DTDTRF

\section{Introduction}

Nowadays, one can observe the increasing use of automation and robotization, which replaces human labor. New applications of industrial robots are widely used especially for repetitive and high precision tasks or monotonous activities demanding physical exertion (e.g. welding, handling). Industrial robots have mobility similar to human arms and can perform various complex actions like a human, but they do not get tired and bored. In addition, they have much greater reliability then human operators. The problem of industrial robot reliability is like machine reliability and is well known and described in the literature, but because of the complexity of robotic systems is also much more complex and is connected with safety requirements and specific robot related problems (near failure situations, hardware failures, software failures, singularity, human errors etc.). Safety is very important, becausethere were many accidents at work with robots involved, and some of them were deadly. Accidents were caused rather more often by human errors than by failures of the robots.

The research about robot reliability was started in 1974 by Engleberger, with publication, which is a summary of three million hours of work of the first industrial robots-Unimate[1]. A very comprehensive discussion over the topic is presented by Dhillon in the book, which covers the problems of robot reliability and safety, including mathematical modelling of robot reliability and some examples[2]. An analysis of publications on robot reliability up to 2002 is available in Ref. Dhillon et al.[3], and some of the important newer publications on robot reliability and associated areas are listed in the book [4].The modern approach to reliability and safety of the robotic system is presented in the book, which includes Robot Reliability Analysis Methods and Models for Performing Robot Reliability Studies and Robot Maintenance[5]. The reliability is strongly connected with safety and productivity, therefore other researches include the design methods of a safe cyber physical industrial robotic manipulator and safety-function design for the control system or simulation method for human and robot related performance and reliability[6-7]. There are fewer publications about the real robot reliability and about occurring failures [8]. The surveyshows that only about 50 percent of robot users have monitoring and collect data about robot failures.

Failure analysis of approximately 200 mature robots in automated production lines, collected from automotive applications in the UK from 1999, is presented in the article, considering Pareto analysis of major failure modes. However, presented data did not reveal sufficiently fine detail of failure history to extract good estimates of the robot failure rate[9-10].

In the article11. Sakai et al.[11], the results of research about robot reliability at Toyota factory are presented. The defects of 300 
units of industrial robots in a car assembly line were analyzed, and a great improvement in reliability has been achieved. The authors consider as significant activities that have been driven by robot users who are involved in the management of the production line. Nowadays, robot manufacturers declare very high reliability of their robots [12]. The best reliability can be achieved by the robots with DELTA and SCARA configuration. This is connected with lower number of links and joints, compared to other articulated robots. Because each additional link with serial connection causes an increase of the unreliability factors, therefore, some components are connected parallel, especially in the Safety Related Part of the Control System (SRP/CS), which have doubled number of some elements, for example emergency stops. Robots are designed in such way that any single, reasonably foreseeable failure will not lead to the robot's hazardous motion [13].Modern industrial robots are designed to be universal manipulating machines, which can have different sort of tools and equipment for specific types of work. However, the robot's equipment is often custom made and may turn out to be unreliable as presented in, therefore, the whole robotic system requires periodic maintenance, following to the manufacturer's recommendations [14-15].

operators and robots in cooperative tasks, therefore, the safety plays a key role. Safety can be transposed in terms of functional safety addressing the functional reliability in the design and implementation of devices and components that build the robotic system [16].

\section{Robot Reliability}

The reliability of objects such as machines or robots is defined as the probability that they will work correctly for a given time under defined working conditions. The general formula for obtaining robot reliability is [2]:

$$
R_{r}(t)=\exp \left(-\int_{0}^{t} \lambda_{r}(t) d t\right)
$$

Where:

$\mathrm{R}_{\mathrm{r}}(\mathrm{t})$ is the robot reliability at time $\mathrm{t}$,

$\lambda_{\mathrm{r}}(\mathrm{t})$ is the robot failure rate.

In practice, for description of reliability, in most cases the MTTF (Mean Time to Failure) parameter is used, which is the expected value of exponentially distributed random variable with the failure rate $\lambda \mathrm{r}[2]$.

$$
\text { MTTF }=\int_{0}^{\infty} R_{r}(t) d t=\int_{0}^{\infty} e^{-\lambda_{r} t} d t=\frac{1}{\lambda_{r}} .
$$

In real industrial environments, the following formula can be used to estimate the average amount of productive robot time, before robot failure [2]:

$$
M T T F=\frac{P H R-D T D T R F}{N R F}
$$

Where:

PHR - is the production hours of a robot,

$\mathrm{NRF}$ - is the number of robot failures,

DTDTRF - is the downtime due to robot failure in hours,

MTTF - is the robot mean time to failure.

In the case of repairable objects, the MTBF (Mean Time Between Failures), and the MTTR (Mean Time to Repair) parameters, can be used.

$$
M T B F=M T T F+M T T R
$$

The reliability of the robotic system depends on the reliability of its components. The complete robotic workstation includes:
A. Manipulation unit (robot arm),
B. controller (computer with software),
C. equipment (gripper, tools),

D. workstation with workpieces and some obstacles in the robot working area,

E. safety system (barriers, curtains, sensors),

F. human operator (supervising, set up, teaching, maintenance).

The robot system consists of some subsystems that are serially connected (as in the Figure 1) and have interface for communication with the environment or teaching by the human operator.The robot arm can have different number of links and joints $\mathrm{N}$. Typical articulated robots have $\mathrm{N}=5-6$ joints as in the Figure 2, but more auxiliary axes are possible.

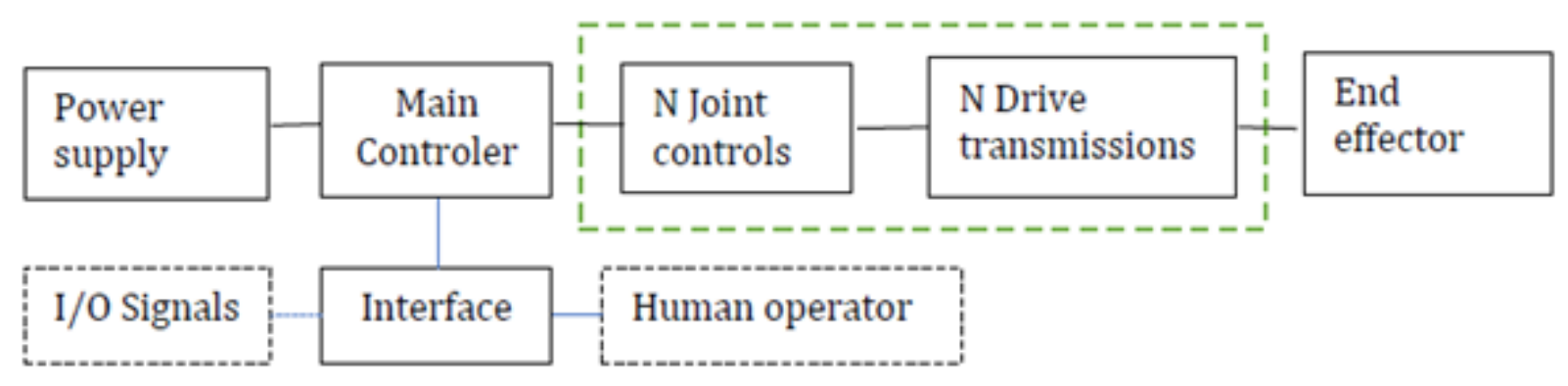

Figure 1: The schema of industrial robot's structure with main subsystems. 


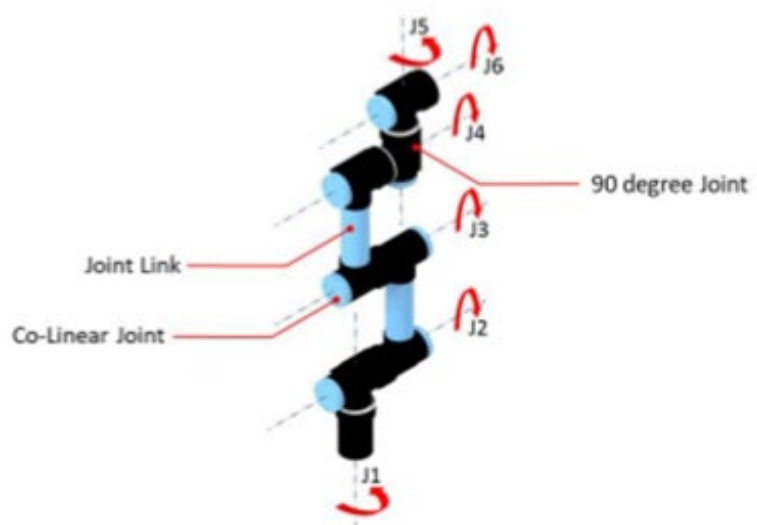

Figure 1: The schema of industrial robot's structure with main subsystems.

For serially connected subsystems, each failure of one component brings the whole system to fail. Considering complex systems, consisting of $n$ serially linked objects, each of which has exponential failure times with rates $\lambda_{i}, i=1,2, \ldots, n$, the resultant overall failure rate $\lambda_{S}$ of the system is the sum of the failure rates of each element $\lambda_{i}[2]$ :

$$
\lambda_{s}=\sum_{i=1}^{n} \lambda_{i}
$$

Moreover, the system MTBFS is the sum of inverse $\mathrm{MTBF}_{i}$, of linked objects:

$$
\frac{1}{M T B F_{s}}=\sum_{i=1}^{n} \frac{1}{M T B F_{i}}
$$

There are different types of failures possible:
A. Internal hardware failures (mechanical unit, drive, gear)

B. Internal software failures (control system),

C. External component failures (equipment, sensors, wiring),

D. Human related errors and failures that can be:

$$
\begin{aligned}
& \text { a. Dangerous for humans (e.g. unexpected robot } \\
& \text { movement), } \\
& \text { b. Non-dangerous, fail-safe (robot unable to } \\
& \text { move). }
\end{aligned}
$$

Also possible are near failure situations and robot related problems, which require the robot to be

stopped and human intervention is needed (e.g. recalibration, reprograming).Because machinery failures may cause severe disturbances in production processes, the availability of means of production plays an important role for insuring the flow of production. Inherent availability can be calculated with the formula 7 [2].

$$
\text { Availablity }=\frac{M T B F}{M T B F+M T T R}
$$

For example, the availability of Unimate robots was about 98 $\%$ over the 10 -years period with $\mathrm{MTBF}=500 \mathrm{~h}$ and $\mathrm{MTTR}=8$ hours [2].

The reliability of the first robot generation represents the typical bathtub curve (as in Figure3), with high rate of early "infant mortality" failures, the second part with a constant failure rate, known as random failures and the third part is an increasing failure rate, known as wear-out failures (it can be described with the Weibull distribution).

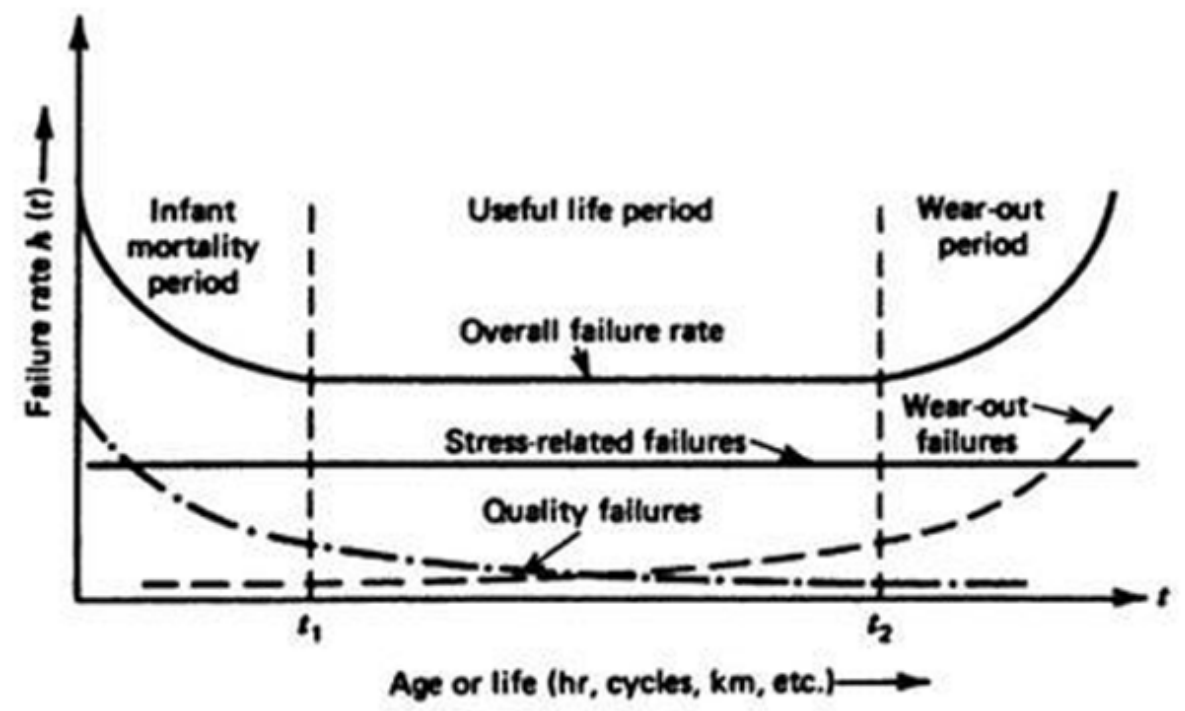

(b)

Figure 3: The failure rate of the first robot generation [18] 
Therefore, the standard [17] was provided, in order to minimize testing requirements that will qualify a newly manufactured (or a newly rebuilt industrial robot) to be placed into use without additional testing. The purpose of this standard is to provide assurance, through testing, that infant mortality failures in industrial robots have been detected and corrected by the manufacturer at their facility prior to shipment to a user. Because of this standard, the next robot generation has achieved better reliability, without early failures, with MTBF about 8000 hours [16].In the articleSakai\&Amasaka[11], the results of research about robot reliability at Toyota are presented. Great improvement was achieved with an increase of the MTBF to about 30000 hours.

Nowadays, robot manufacturers declare an average of MTBF $=$ $50,000-60,000$ hours or 20 - 100 million cycles of work [12]. The best reliability is achieved by the robots with SCARA and DELTA configuration. This is connected with lower number of links and joints, compared to other articulated robots.Some interesting conclusions from the survey about industrial robots conducted in Canada in year 2000 are as follows [9]:

A. Over 50 percent of the companies keep records of the robot reliability and safety data,

B. In robotic systems, major sources of failure were software failure, human error and circuit board troubles from the users' point of view,

C. Average production hours for the robots in the Canadian industries were less than 5,000 hours per year,

D. The most common range of the experienced MTBF was 500-1000h (from the range 500-3000h)

E. Most of the companies need about 1-4h for the MTTR of their robots (but also in many cases the time was greater than $10 \mathrm{~h}$ or undefined).

The current industrial practice show that the most unreliable components are in the robot's equipment, including grippers, tools, sensors, wiring, which are often custom made for different purposes. This equipment can be easily repaired by the robot user's repair department. But the failure of critical robot component requires intervention of the manufacturer service and can take much more time to repair (and can be counted in days). Therefore, for better performance and reliability of the robotic system, periodic maintenance is recommended.

\section{Robot Maintenance}

Three basic types of maintenance for robots used in industry are as follows [4]:

\section{Preventive maintenance}

This is basically concerned with servicing robot system components periodically (e.g. daily, yearly. ...)

\section{Corrective maintenance}

This is concerned with repairing the robot system whenever it breaks down.

\section{Predictive maintenance}

Nowadays, many robot systems are equipped with sophisticated electronic components and sensors; some of them are capable of being programmed to predict when a failure might happen and to alert the concerned maintenance personnel (e.g. self-diagnostic, singularity detection).Robot maintenance should be performed, following to the robot manufacturer's recommendations, which are summarized in the Table 1[15]. Preventive maintenance should be provided before each automatic run, including self-diagnostic of the robot control system, visual inspection of cables and connectors, checking for oil leakage or abnormal signals like noise or vibrations. The replacement of the battery, which powers the robot's positional memory, is needed yearly. If the memory is lost, then remastering (recalibration, synchronization) is needed.Replenishing the robot with grease every recommended period is needed to prevent the mechanical components (like gears) from wearing out. Special greases are used for robots (e.g. Moly White RE No.00) or grease dedicated for specific application like for the food-industry. Every 3-5 years a fully technical review (overhaul) with replacement of filters, fans, connectors, seals, etc. is recommended.

Table 1: Robot maintenance procedures $[4,15]$.

\begin{tabular}{|c|c|c|}
\hline No & Maintenance Procedure & Time Period \\
\hline 1 & Preventive maintenance-visual inspection, checking of abnormal signals, self-diagnostic of control system & daily \\
\hline 2 & Periodic maintenance service-battery replacement & yearly \\
\hline 3 & Overhaul-greasing, replacement of filters, fans, etc. & every $3 \div 5$ years \\
\hline 4 & Refurbishment-cleaning, replacement of defected or damaged components, e.g. bearings, brakes, gears, motors & after $10 \div 15$ years \\
\hline
\end{tabular}

Performing daily inspection, periodic inspection, and maintenance can keep the performance of robots in a stable state for a long period. The lifecycle of typical robot is about 10-15 years, because the wear of key mechanical components (drives, gears, bearings, brakes) causes backlash and positional inaccuracy. After that time a refurbishment of the robot is possible, and it can work further for long time. Refurbished Robots are also called remanufactured, reconditioned, or rebuilt robots.

\section{Conclusion}

Nowadays modern industrial robots have achieved high reliability and functionality; therefore, they are widely used. This 
is confirmed by more than one and half million of robots working worldwide. According to the probability theory, in such large robot population the failures of some robots are almost inevitable. The failures are random, and we cannot predict exactly where and when, they will take place. Therefore, the robot users should be prepared and should undertake appropriate maintenance procedures. This is important, because industrial robots can highly increase the productivity of manufacturing systems, compared to human labor, but every robot failure can cause severe disturbances in the production flow, therefore periodic maintenance is required, in order to prevent robot failures. High reliability is also important for the next generation of collaborative robots, which should work close to human workers, and safety must be guaranteed without barriers. Also, some sorts of service robots, which should help nonprofessional people (e.g. health care of disabled people) must have high reliability and safety. There have already been some accidents at work, with robots involved, therefore, the next generation of intelligent robots should be reliable enough to respect the Asimov's laws and do not hurt people, even if they make errors and give wrong orders.

\section{References}

1. Engleberger JF (1974) Three Million Hours of Robot Field Experience. The Industrial Robot 4(1): 164-168.

2. Dhilon BS (1991) Robot Reliability and Safety. Springer Verlag, New York, USA

3. Dhillon BS, Fashandi ARM, Liu KL (2002) Robot systems reliability and safety: a review. Journal of Quality in Maintenance Engineering 8(3) :170-212

4. Dhillon BS (2007) Applied Reliability and Quality. Fundamentals, Methods and Procedures. Springer Verlag, New York, USA

5. Dhilon BS (2015) Robot System Reliability and Safety: A Modern Approach. CRC Press, USA
6. Francalanza E, Fenech A, Cutajar P (2018) Design of a Cyber Physical Industrial Robotic Manipulator. Robot Autom Eng J 2(4): 555594.

7. Lee S, Yamada Y, Ichikawa K, Matsumoto O, Homma K, et al. (2014) Safety-Function Design for the Control System of a Human-Cooperative Robot Based on Functional Safety of Hardware and Software. IEEEASME Transactions on Mechatronics 19(2): 719-729.

8. Gołda G, Kampa A, Paprocka I (2018) Analysis of Human Operators and Industrial Robots Performance and Reliability. MPER 9(1): 24-33

9. Dhillon BS, Aleem MA (2000) A report on robot reliability and safety in Canada: a survey of robot users. Journal of Quality in Maintenance Engineering. 6(1): 61-74.

10. Starr AG, Wynne RJ, Kennedy I (1999) Failure analysis of mature robots in automated production. Journal of Engineering Manufacture 213 (8): 813-824.

11. Sakai H, Amasaka K (2007) The robot reliability design and improvement method and the advanced Toyota production system. Industrial Robot: An International Journal 34(4): 310-316.

12. Hägele M, Nilsson K, Pires JN (2008) Industrial Robotics, in Springer Handbook of Robotics. Springer, Berlin

13. ANSI/RIA R15.06-2012 American National Standard for Industrial Robots and Robot Systems- Safety Requirements.

14. Barosz P, Gołda G, Kampa A (2018) Productivity analysis of manufacturing line with industrial robots and human operators. Industrial Robot: An International Journal.

15. FANUC Robotics. Maintenance Manual, 2001

16. Pedrocchi N, Vicentini F, Matteo M, Tosatti LM (2013) Safe Human Robot Cooperation in an Industrial Environment. International Journal of Advanced Robotic Systems Pp. 1-13.

17. ANSI/RIA R15.05-3-1992 (R1999) Industrial Robots and Robot Systems - Reliability Acceptance Testing-Guidelines.

18. Nof Y (1999) Handbook of industrial robotics. John Wiley \& Sons, New York, USA.

\section{Your next submission with Juniper Publishers will reach you the below assets}

- Quality Editorial service

- Swift Peer Review

- Reprints availability

- E-prints Service

- Manuscript Podcast for convenient understanding

- Global attainment for your research

- Manuscript accessibility in different formats

( Pdf, E-pub, Full Text, Audio)

- Unceasing customer service

Track the below URL for one-step submission https://juniperpublishers.com/online-submission.php 